\title{
Peritoneal Cancer Index
}

National Cancer Institute

\section{Source}

National Cancer Institute. Peritoneal Cancer Index. NCI Thesaurus. Code C123361.

A method for detailed evaluation of the peritoneal spread of tumors of gastrointestinal origin. The index is based on tumor size in 13 separate regions, 9 regions in the abdomen and 4 in the small intestine. Each region is given a score from 0 to 3, based on the size of any tumors present, and the cancer index is the sum of all scores, ranging from 0 to 39. This score has been found to be a prognostic indicator of survival. 